ЧАСТОТА И СТРУКТУРА МЕТАБОЛИЧЕСКИХ НАРУШЕНИЙ У ЖЕНЩИН С ОЖИРЕНИЕМ В СОЧЕТАНИИ С СИНДРОМОМ ПОЛИКИСТОЗНЫХ ЯИЧНИКОВ

\author{
(с Е.В. Болотова, А.В. Дудникова*, В.А. Крутова, Н.С. Просолупова
}

Кубанский государственный медицинский университет, Краснодар, Россия

Обоснование. Ожирение считается мировой эпидемией и является одной из значимых медико-социальных проблем. Исследования последних лет показывают, что в 25-45\% случаев при ожирении выявляется синдром поликистозных яичников (СПКЯ). Остается спорным вопрос о влиянии ожирения на патогенез метаболических нарушений у данной категории пациенток.

Цель. Определить частоту и структуру метаболических нарушений у женщин с ожирением в сочетании с синдромом поликистозных яичников (СПКЯ).

Методы. Проведено одноцентровое поперечное выборочное обследование женщин репродуктивного возраста. В рамках исследования проводилась клиническая антропометрия с измерением массы тела, роста, окружности талии (ОТ) и бедер (ОБ), последующим расчетом индекса массы тела (ИМТ) и соотношения ОТ/ОБ, измерение артериального давления (АД). Проведено биохимическое исследование крови, исследован гормональный статус, выполнено ультразвуковое исследование органов малого таза.

Результаты. Проведено обследование 136 женщин репродуктивного возраста. В группу 1 включены женщины с ожирением без СПКЯ (59 пациенток), в группу 2 - женщины с ожирением в сочетании с СПкЯ (45 пациенток), в контрольную группу - 38 здоровых женщин. Среди пациенток 2-й группы статистически значимо чаще встречались дислипидемия, висцеральное ожирение, артериальная гипертензия (АГ), инсулинорезистентность, гиперинсулинемия, гиперурикемия $(p<0,05)$. Выявлены корреляции между ИМТ и триглицеридами, тестостероном и общим холестерином (OXC) (p<0,05). Пациентки с ожирением и СПКЯ имели статистически значимо более высокий уровень С-реактивного белка (СРБ), фибриногена, антимюллерова гормона, печеночных трансаминаз (р<0,05). Дефицит витамина D выявлен у 13,2\%, недостаточность - у 22,7\% пациенток. Отмечена статистически значимая корреляция между уровнем 25(OH)D и показателями ИМТ, уровнями фолликулостимулирующего, лютеинизирующего ( $<<0,05)$, антимюллерова гормонов $(\mathrm{p}=0,008)$.

Заключение. Выявлена высокая частота метаболических нарушений у женщин с ожирением в сочетании с СПкЯ, что обуславливает необходимость проведения раннего скрининга, диагностики и лечения этих нарушений для укрепления репродуктивного здоровья и профилактики хронических неинфекционных заболеваний.

КЛЮЧЕВЫЕ СЛОВА: ожирение; синдром поликистозных яичников; метаболические нарушения уженщин.

\title{
THE FREQUENCY AND STRUCTURE OF METABOLIC DISORDERS IN OBESE WOMEN IN COMBINATION WITH POLYCYSTIC OVARY SYNDROME
}

\author{
(c) Elena V. Bolotova, Anna V. Dudnikova*, Victoria A. Krutova, Natalia S. Prosolupova
}

Kuban state medical university, Krasnodar, Russia

BACKGROUND: Obesity is considered a global epidemic and is one of the most significant medical and social problems. Research in recent years shows that in $25-45 \%$ of cases of obesity, polycystic ovary syndrome (PCOS) is detected. The influence of obesity on the pathogenesis of metabolic disorders in this category of patients remains controversial

AIMS: to determine the frequency and structure of metabolic disorders in obese women in combination with polycystic ovary syndrome (PCOS).

MATERIALS AND METHODS: A single-center cross-sectional sample survey of women of reproductive age was conducted. The study included clinical anthropometry with measurement of body weight, height, waist circumference (OT) and hip circumference (OB), followed by calculation of body mass index (BMI) and OT/OB ratio, and measurement of blood pressure (BP). A biochemical blood test was performed, the hormonal status was examined, and an ultrasound examination of the pelvic organs was performed.

RESULTS: A survey of 136 women of reproductive age was conducted. Group 1 included obese women without PCOS (59 patients), group 2 included obese women in combination with PCOS (45 patients), and the control group included 38 healthy women. Group 1 included obese women without PCOS (59 patients), and group 2 included obese women with PCOS (45 patients), in the control group - 38 healthy women. Among group 2 patients, dyslipidemia, visceral obesity, arterial hypertension, insulin resistance, hyperinsulinemia, and hyperuricemia were significantly more common $(p<0,05)$. Correlations between BMI and triglycerides, testosterone and total cholesterol were found $(p<0,05)$. Obese and PCOS patients had significantly higher levels of C-reactive protein(CRP), fibrinogen, anti-Müllerian hormone (AMH), and hepatic transaminases 
$(p<0,05)$. Vitamin D deficiency was detected in $13,2 \%$ of patients, and insufficiency - in $22,7 \%$ of patients. There is a statistically significant correlation between the level of $25(\mathrm{OH}) \mathrm{D}$ and indicators of BMI, follicle-stimulating hormone, luteinizing hormone $(p<0,05)$, the level of $A M H(p=0,008)$.

CONCLUSIONS: A high frequency of metabolic disorders in obese women in combination with PCOS has been identified, which necessitates early screening, diagnosis and treatment of these disorders to strengthen reproductive health and prevent chronic non-communicable diseases.

KEYWORDS: obesity; polycystic ovary syndrome; metabolic disorders in women.

\section{ОБОСНОВАНИЕ}

Ожирение по праву считается мировой эпидемией и является одной из значимых медико-социальных проблем [1]. Его основным механизмом, согласно определению ВОЗ, является хронический калорийный дисбаланс между потреблением пищи и расходом энергии [1]. В настоящее время доказано влияние величины индекса массы тела (ИМТ) на риск развития хронических неинфекционных заболеваний [1]. Исследования последних лет выявили взаимосвязь бесплодия с высоким ИМТ в 25-50\% случаев [2]. Установлена прямая зависимость между ИМТ и абдоминальным ожирением, увеличением ИМТ и тяжестью нарушений овариальной функции, сопровождающихся ановуляцией, неполноценностью лютеиновой фазы цикла и, соответственно, уменьшением числа беременностей [1-3]. В 25-45\% случаев при ожирении выявляется синдром поликистозных яичников (СПКЯ); в современных работах имеются указания на серьезные неблагоприятные долгосрочные эффекты ожирения в виде сердечно-сосудистых, метаболических и репродуктивных нарушений $[2,4]$.

Зачастую СПКЯ сопровождается хронической ановуляцией, андроидным типом ожирения, гиперинсулинемией $[2,4]$. Вместе с тем не у всех женщин с ожирением диагностируют СПКЯ и не все пациентки с СПКЯ имеют ожирение; то есть ожирение, независимо от СПКЯ, ассоциируется с ановуляцией $[5,6]$. В настоящее время имеется ограниченная информация о метаболических особенностях женщин с СПКЯ, остается спорным вопрос о влиянии ожирения на патогенез данных метаболических нарушений у данной категории пациенток.

\section{ЦЕЛЬ}

Определить частоту и структуру метаболических нарушений у женщин с ожирением в сочетании с СПКЯ.

\section{МЕТОДЫ}

Место и время проведения исследования

Место проведения. Исследование проводилось на базе клиники ФГБОУ ВО КубГМУ МЗ РФ.

Время исследования. С декабря 2019 г. по март 2020 г.

\section{ИЗУЧАЕМЫЕ ПОПУЛЯЦИИ}

Изучались 3 популяции: здоровые женщины репродуктивного возраста, не имеющие ожирения и СПКЯ, женщины, имеющие ожирение, и женщины с ожирением и СПКЯ.

\section{1. Популяция здоровых женщин (контрольная} группа).

Критерии включения: женский пол, возраст 18-45 лет, ИМТ 18-25 кг/M².
Критерии исключения: все критерии исключения для популяции 3, а также наличие СПКЯ.

\section{2. Популяция женщин с ожирением.}

Критерии включения: женский пол, возраст 18-45 лет,

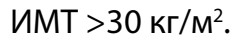

Критерии исключения: все критерии исключения для популяции 3, а также наличие СПКЯ.

\section{3. Популяция женщин с ожирением и СПКя.}

Критерии включения: женский пол, возраст 18-45 лет, ИМТ >30 кг/м², наличие СПКЯ.

Критерии исключения: наличие новообразований любой локализации, синдром Иценко-Кушинга, беременность, лактация, ранняя/хирургическая менопауза, любые хронические заболевания в стадии декомпенсации, наличие сахарного диабета.

Способ формирования выборки из изучаемой популяции (или нескольких выборок из нескольких изучаемых популяций)

Выборка формировалась произвольным способом на основании обследования, включавшего сбор анамнеза, определение антропометрических и лабораторных показателей в соответствии с заданными критериями.

\section{Дизайн исследования}

Проведено одноцентровое интервенционное одномоментное трехвыборочное сравнительное исследование.

\section{Описание медицинского вмешательства}

Обследование пациенток включало сбор анамнеза, физикальный осмотр с измерением антропометрических (возраст, пол, рост, масса тела, окружность талии (ОТ), окружность бедер (ОБ), расчет ИМТ) и клинических (артериальное давление (АД), частота сердечных сокращений (ЧСС)) параметров.

Участницы исследования сдавали образцы крови в утренние часы натощак, на 2-5-й день менструального цикла или в любой день в отсутствие менструации. Исследовали стандартный биохимический профиль, общеклинический анализ крови и мочи, липидный состав крови (общий холестерин (ОХС), триглицериды (ТГ), липопротеины низкой плотности (ЛПНП), липопротеины высокой плотности (ЛПВП); тиреотропный гормон (ТТГ), антитела к тиреопероксидазе (АТТП), свободный Т4 (СТ4), уровень 25(OH)D, инсулин, эстрогены, прогестерон, фолликулостимулирующий гормон (ФСГ), лютеинизирующий гормон (ЛГ), тестостерон, глобулин, связывающий половые стероиды (ГСПГ), антимюллеров гормон (АМГ)).

Ультразвуковое исследование (УЗИ) органов малого таза проводилось на 2-3-й день менструального цикла для расчета количества антральных фолликулов и объема яичников. 


\section{Основной исход исследования}

Основными оцениваемыми параметрами в рамках исследования явились: липидный профиль, уровень мочевой кислоты, С-реактивный белок (СРБ), наличие висцерального ожирения, артериальной гипертензии (АГ), инсулинорезистентности.

\section{Дополнительные исходы исследования}

Дополнительные оцениваемые параметры: уровень креатинина с расчетом скорости клубочковой фильтрации (СКФ).

\section{Методы}

Лабораторные исследования выполнены на гематологическом анализаторе MEK 8222 (Nihon Kohden, Япония), автоматическом анализаторе для клинической биохимии SAPPHIRE 400 (Hirose Electronic System, Япония), иммунохимическом анализаторе Roche Cobas e 411 (Швейцария). По уровню ОХС различали умеренную (5,0<ОХС <6,5 ммоль/л) и выраженную (ОХС >6,5 ммоль/л) гиперхолестеринемию, коэффициент атерогенности (КА) определяли по формуле:

$$
\text { КА }=\frac{\text { ХС ЛПНП-ХС ЛПОНП }}{\text { ХС ЛПВП }} \text { [4]. }
$$

Для каждой пациентки рассчитывали индекс инсулинорезистентности HOMA-IR (Homeostasis Model Assessment of Insulin Resistance) по формуле: инсулин (мкЕд/мл)×глюкоза (ммоль,л)/22,5. При уровне инсулина $\geq 18,0$ мкЕд/мл диагностировали гиперинсулинемию, при величине показателя НОМА >3,39 - инсулинорезистентность (ИР) [5]. У всех пациенток в исследовании гиперандрогения была подтверждена биохимически на основании расчета индекса свободных андрогенов (ИСА), определяемого по формуле: общий тестостерон, нмоль/л/ГСПГ, нмоль/л $\times 100 \%$, нормальное значение ИСА у женщин репродуктивного периода - 0,8-11\% [5]. Согласно рекомендациям Российской ассоциации эндокринологов, дефицит витамина D был определен как значение уровня витамина $D<20$ нг/мл, недостаточность значение $\geq 20$ и <30 нг/мл [7]. Проводилось измерение антропометрических (возраст, пол, рост, масса тела, ОТ, ОБ, расчет ИМТ) и клинических (АД, ЧСС) параметров. ИМТ, кг/м², определялся как масса тела (кг)/рост (м) ${ }^{2}$ [7]. Для диагностики висцерального (абдоминального) ожирения проводилось измерение ОТ по среднеподмышечной линии на середине расстояния между вершиной гребня подвздошной кости и нижним краем последнего ребра. Критерием висцерального ожирения считали ОТ $\geq 80$ см. [8]. АД определяли в положении сидя с использованием полуавтоматического тонометра OMRON (Япония). Оптимальным считали АД до 119/79 мм рт. ст.; нормальным - 120-129/80-84 мм рт. ст., высоким нормальным - 130-139/85-89 мм рт. ст., АГ І ст. - при АД 140-159/90-99мм рт.ст.;АГІІст. - 160-179/100-109мм рт.ст., АГ ІІІ Ст. - при АД >180/110 мм рт. ст. [8].

УЗИ органов малого таза проводили на аппарате экспертного класса Volusson с использованием трансвагинального конвексного датчика 5-9 МГц: измеряли длину, переднезадний и поперечный размеры матки, оценивали состояние миометрия и эндометрия, измеряли размеры яичников, изучали их структуру (количество фол- ликулов в одном срезе). Диагноз СПКЯ устанавливали на основании Роттердамского консенсуса европейских экспертов (2003 г.): наличие любых 2 из 3 следующих признаков: 1 - нерегулярные менструации ( $\leq 6 /$ год), олиго- или ановуляция; 2 - симптомы избыточной секреции и активности андрогенов (акне, гирсутизм) и/или биохимическая гиперандрогения; 3 - типичные изменения яичников по данным УЗИ ( $\geq 12$ фолликулов размером 2-9 мм), если исключены другие причины, которые могут вызвать СПКЯ [4].

\section{Статистический анализ}

Принципы расчета размера выборки: размер выборки предварительно не рассчитывался.

Методы статистического анализа данных. Статистическая обработка проведена с использованием пакета статистической программы Excel 2013 совместно с надстройкой AtteStat 12.0.5. С целью выбора метода статистического анализа проводилось определение нормальности распределения. Проверку значимости различий между средними в разных группах проводили с помощью дисперсионного анализа (ANOVA — Analysis of Variation). Оценку статистической значимости различий двух или нескольких относительных показателей (частот, долей) выполняли при помощи критерия $\mathrm{X}^{2}$ Пирсона. Корреляционный анализ для уточнения взаимосвязи показателей проведен с использованием коэффициента корреляции Пирсона (r). Показатели представлены в виде M (SD), где M - среднее значение, SD - стандартное отклонение. Корреляционные связи и различия сравниваемых показателей считали статистически значимыми при $p<0,05$.

\section{Этическая экспертиза}

Протокол исследования был одобрен локальным этическим комитетом ФГБОУ ВО КубГМУ (протокол заседания №83 от 07.12.2019). До включения в исследование у всех пациентов получено письменное информированное согласие.

\section{РЕЗУЛЬТАТЬ}

Объекты (участники) исследования

В исследование были включены 136 пациенток в возрасте от 18 до 45 лет, наблюдавшихся в Клинике ФГБОУ ВО КубГМУ МЗ РФ по поводу планирования беременности. Соответственно критериям пациентки разделены на 3 группы: в группу 1 включены женщины с ожирением без СПКЯ (59 пациенток), в группу 2 - женщины с ожирением в сочетании с СПКЯ (45 пациенток), в контрольную группу - 38 здоровых женщин.

\section{Основные результаты исследования}

Клинико-лабораторные данные обследованных пациенток представлены в таблице 1.

Наиболее высокие средние уровни ИМТ, ОХС, АД были зарегистрированы в группе пациенток с ожирением в сочетании с СПКЯ, однако статистически значимой разницы по сравнению с 1-й группой не выявлено (р>0,05). Получены статистически значимые различия по ИМТ между 2-й и контрольной группами $(p=0,045)$. Все пациентки 1-й и 2-й групп имели ожирение; вместе 
Таблица 1. Клинико-лабораторная характеристика пациентов

\begin{tabular}{|c|c|c|c|c|}
\hline \multirow[b]{3}{*}{ Показатели } & \multicolumn{4}{|c|}{$n=136$} \\
\hline & $\begin{array}{c}1 \text { группа } \\
\text { с ожирением без } \\
\text { СПкя }\end{array}$ & $\begin{array}{c}2 \text { группа } \\
\text { с ожирением и } \\
\text { СПкя }\end{array}$ & группа контроля & $\mathbf{p}$ \\
\hline & $n=59$ & $n=45$ & $n=32$ & \\
\hline Возраст, лет & $26,61 \pm 4,83$ & $27,12 \pm 5,09$ & $25,45 \pm 4,16$ & $p=0,479$ \\
\hline ИМТ, кг/м² & $30,24 \pm 3,72$ & $33,05 \pm 4,82$ & $22,51 \pm 3,23$ & $p=0,073$ \\
\hline ОТ/ОБ & $0,79 \pm 0,06$ & $1,22 \pm 0,09$ & $0,66 \pm 0,04^{1}$ & $p^{1}=0,001$ \\
\hline АД систолическое, мм рт. ст. & $132,78 \pm 9,41$ & $133,78 \pm 6,45$ & $112,74 \pm 5,41$ & $p=0,657$ \\
\hline АД диастолическое, мм рт. ст. & $74,18 \pm 4,32$ & $78,11 \pm 6,36$ & $72,34 \pm 3,22$ & $p=0,421$ \\
\hline Инсулин, мкМЕ/мл & $14,55 \pm 3,23$ & $18,54 \pm 5,37$ & $13,55 \pm 2,13$ & $p=0.391$ \\
\hline Глюкоза, ммоль/л & $4,92 \pm 0,96$ & $5,68 \pm 1,42$ & $4,62 \pm 0,71$ & $p=0,506$ \\
\hline Индекс HOMA-IR & $2,38 \pm 0,61$ & $4,21 \pm 0,49^{1,2}$ & $2,11 \pm 0,07$ & $\begin{array}{l}p^{1}=0,001 \\
p^{2}=0,02\end{array}$ \\
\hline АМГ, нг/мл & $2,48 \pm 0,31$ & $10,45 \pm 3,12^{1,2}$ & $4,68 \pm 0,291$ & $\begin{array}{l}p^{1}=0,045 \\
p^{2}=0,01\end{array}$ \\
\hline ОХС, ммоль/л & $4,11 \pm 1,09$ & $7,12 \pm 1,23^{1}$ & $3,42 \pm 0,99$ & $p^{1}=0,02$ \\
\hline Триглицериды, ммоль/л & $1,26 \pm 0,18$ & $1,69 \pm 0,78$ & $1,12 \pm 0,23$ & $p=0,485$ \\
\hline ЛПНП, ммоль/л & $3,11 \pm 0,53$ & $3,88 \pm 0,98$ & $2,05 \pm 0,13$ & $p=0,068$ \\
\hline ЛПВП, ммоль/л & $0,97 \pm 0,28$ & $0,67 \pm 0,14^{1}$ & $1,17 \pm 0,13$ & $p^{1}=0,02$ \\
\hline $\mathrm{KA}$ & $2,54 \pm 0,94$ & $4,06 \pm 0,18^{1}$ & $2,01 \pm 0,16$ & $p^{1}=0,001$ \\
\hline Креатинин, мкмоль/л & $84,34 \pm 5,69$ & $91,32 \pm 9,18$ & $71,22 \pm 8,13$ & $p=0,105$ \\
\hline СКФ, мл/мин/1,73 м² & $99,14 \pm 6,17$ & $98,26 \pm 7,79$ & $101,34 \pm 13,11$ & $\mathrm{p}=0,841$ \\
\hline Мочевина, ммоль/л & $4,22 \pm 1,11$ & $5,02 \pm 1,81$ & $4,45 \pm 1,23$ & $\mathrm{p}=0,795$ \\
\hline Мочевая кислота, ммоль/л & $171,13 \pm 13,23$ & $345,56 \pm 31,9^{1,2}$ & $156,13 \pm 12,18$ & $\begin{array}{l}p^{1}=0,001 \\
p^{2}=0,001\end{array}$ \\
\hline Фибриноген, г/л & $4,44 \pm 0,93$ & $6,13 \pm 1,08^{1}$ & $3,34 \pm 0,73$ & $p^{1}=0,035$ \\
\hline СРБ, мг/л & $3,21 \pm 0,08$ & $4,10 \pm 0,45^{1}$ & $0,94 \pm 0,33$ & $p^{1}=0,001$ \\
\hline АЛТ, Ед/л & $20,22 \pm 5,41$ & $42,32 \pm 7,48^{1,2}$ & $14,54 \pm 2,63$ & $\begin{array}{l}p^{1}=0,001 \\
p^{2}=0,02\end{array}$ \\
\hline АСТ, Ед/л & $24,69 \pm 4,89$ & $33,24 \pm 4,89^{1}$ & $14,54 \pm 1,63$ & $\mathrm{p}^{1}=0,003$ \\
\hline Гемоглобин, г/л & $121,23 \pm 7,45$ & $129,13 \pm 6,23$ & $119,13 \pm 4,23$ & $p=0,188$ \\
\hline Тиреотропный гормон, мМе/мл & $2,98 \pm 0,77$ & $3,66 \pm 1,11$ & $3,48 \pm 0,77$ & $p=0,124$ \\
\hline Свободный тироксин, пмоль/л & $18,73 \pm 3,76$ & $15,18 \pm 2,92$ & $16,51 \pm 2,35$ & $p=0,723$ \\
\hline ЛГ, мМед/мл & $6,75 \pm 1,28$ & $12,01 \pm 3,12^{1}$ & $4,75 \pm 0,94^{1}$ & $p^{1}=0,02$ \\
\hline ФСГ, мМед/мл & $6,48 \pm 3,01$ & $4,50 \pm 0,32^{1}$ & $8,79 \pm 2,01^{1}$ & $p^{1}=0,02$ \\
\hline Эстрадиол, пг/мл & $57,97 \pm 9,01$ & $69,12 \pm 10,21$ & $54,87 \pm 8,01$ & $\mathrm{p}=0,275$ \\
\hline Пролактин, нг/мл & $21,65 \pm 4,36$ & $18,52 \pm 6,88$ & $16,55 \pm 4,81$ & $p=0,434$ \\
\hline Прогестерон, нг/мл & $0,72 \pm 0,18$ & $0,59 \pm 0,13$ & $0,49 \pm 0,09$ & $p=0,512$ \\
\hline Тестостерон общий, нмоль/л & $1,49 \pm 0,12$ & $3,37 \pm 0,98^{1,2}$ & $1,09 \pm 0,32$ & $\begin{array}{l}p^{1}=0,03 \\
p^{2}=0,04\end{array}$ \\
\hline ГСПГ, нмоль/л & $29,53 \pm 4,23$ & $22,42 \pm 5,31$ & $43,71 \pm 9,11$ & $\begin{array}{l}p^{1}=0,047 \\
p^{2}=0,291\end{array}$ \\
\hline ИCA, \% & $6,41 \pm 1,25$ & $13,02 \pm 3,32$ & $2,5 \pm 0,09$ & $\begin{array}{l}p^{1}=0,002 \\
p^{2}=0,065\end{array}$ \\
\hline $25(\mathrm{OH}) Д$ & $29,65 \pm 6,5$ & $26,26 \pm 3,13$ & $29,96 \pm 5,13$ & $p=0,539$ \\
\hline
\end{tabular}

Примечание. ${ }^{1}$ - достоверность различий между 2-й и контрольной группами $(p<0,05){ }^{2}$ - достоверность различий между 1-й и 2-й группами. Формат представления данных M $\pm S D$, где M - среднее значение; SD - стандартное отклонение. 
с тем среднее отношение ОТ/ОБ, свидетельствующее о висцеральном ожирении, во 2-й группе было значимо выше $(p=0,04)$. Частота висцерального ожирения регистрировалась чаще в группе пациенток с ожирением и СПКЯ по сравнению с контрольной (100\% vs 40,6\%; $\mathrm{X}^{2}=7,514 ; \mathrm{p}=0,04$ соответственно) (рис. 1). Корреляционный анализ выявил положительную корреляцию средней силы между уровнем сывороточного тестостерона и XC (r=0,305; $p=0,045)$ (рис. 2), между уровнем ТГ и ИМТ $(r=0,347 ; p=0,02)$ (рис. 3), а также отрицательную средней силы корреляцию между ЛПВП и ИМТ ( $r=-0,361 ; p=0,02)$.

Синдром АГ регистрировался значимо чаще у пациенток с ожирением в сочетании с СПКЯ - 35,5\% по сравнению с пациентками с ожирением - 22,2\% ( $x^{2}=4,254$; $\mathrm{p}=0,002)$ и контрольной группы - 6,2\% $\left(\mathrm{x}^{2}=6,354\right.$; $\mathrm{p}=0,001)$ (см. рис. 1).

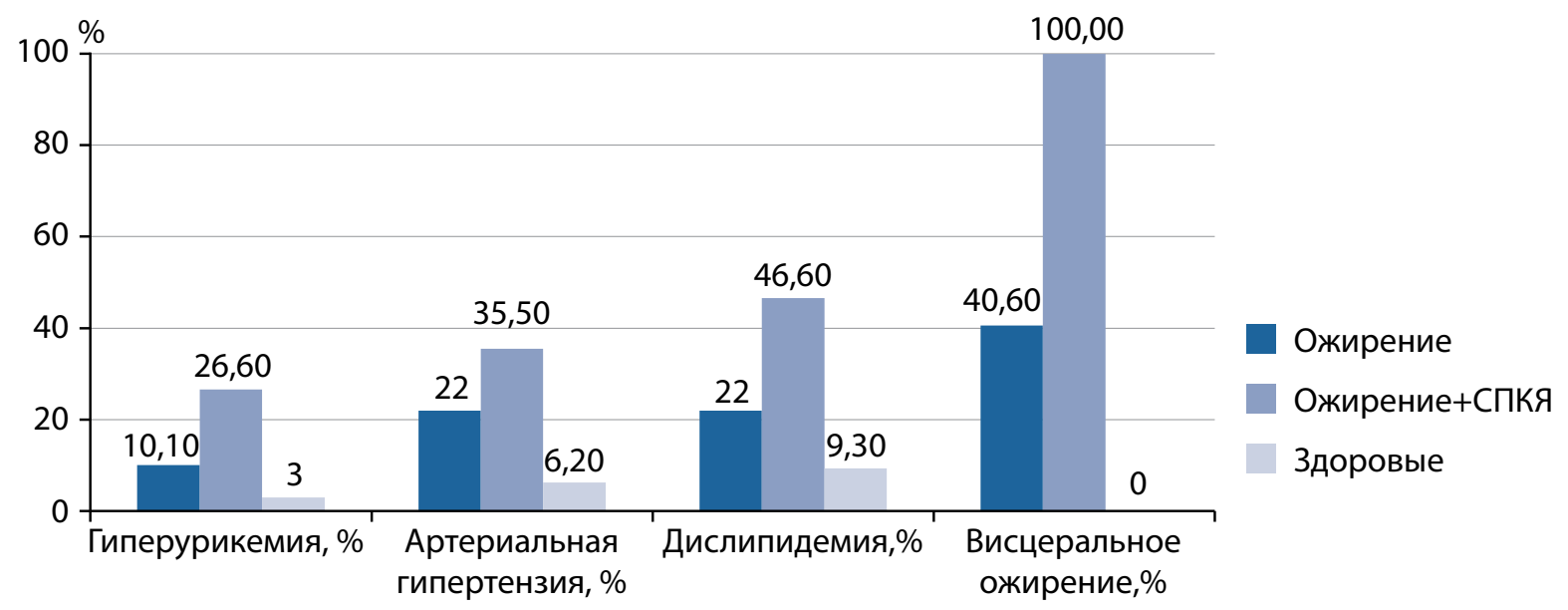

Рисунок 1. Частота метаболических нарушений и артериальной гипертензии в исследуемых группах.

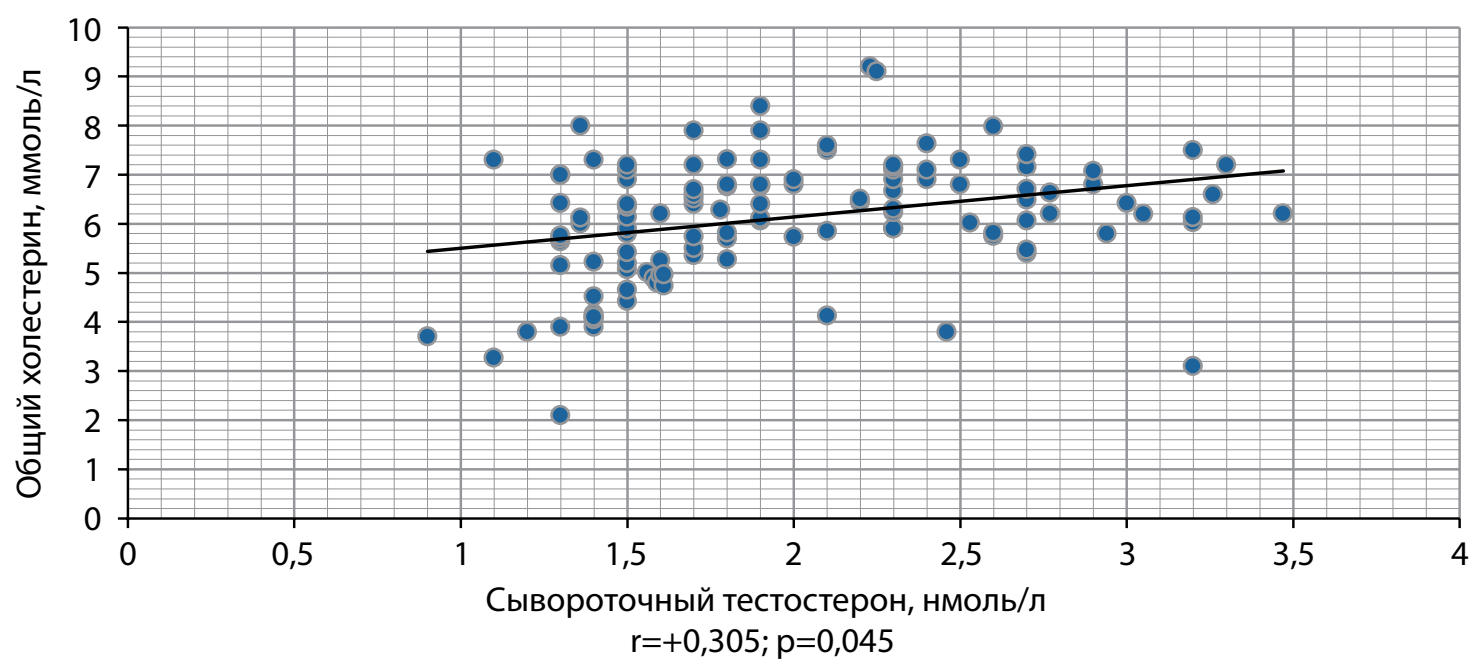

Рисунок 2. График корреляционной зависимости между уровнем сывороточного тестостерона и общего холестерина.

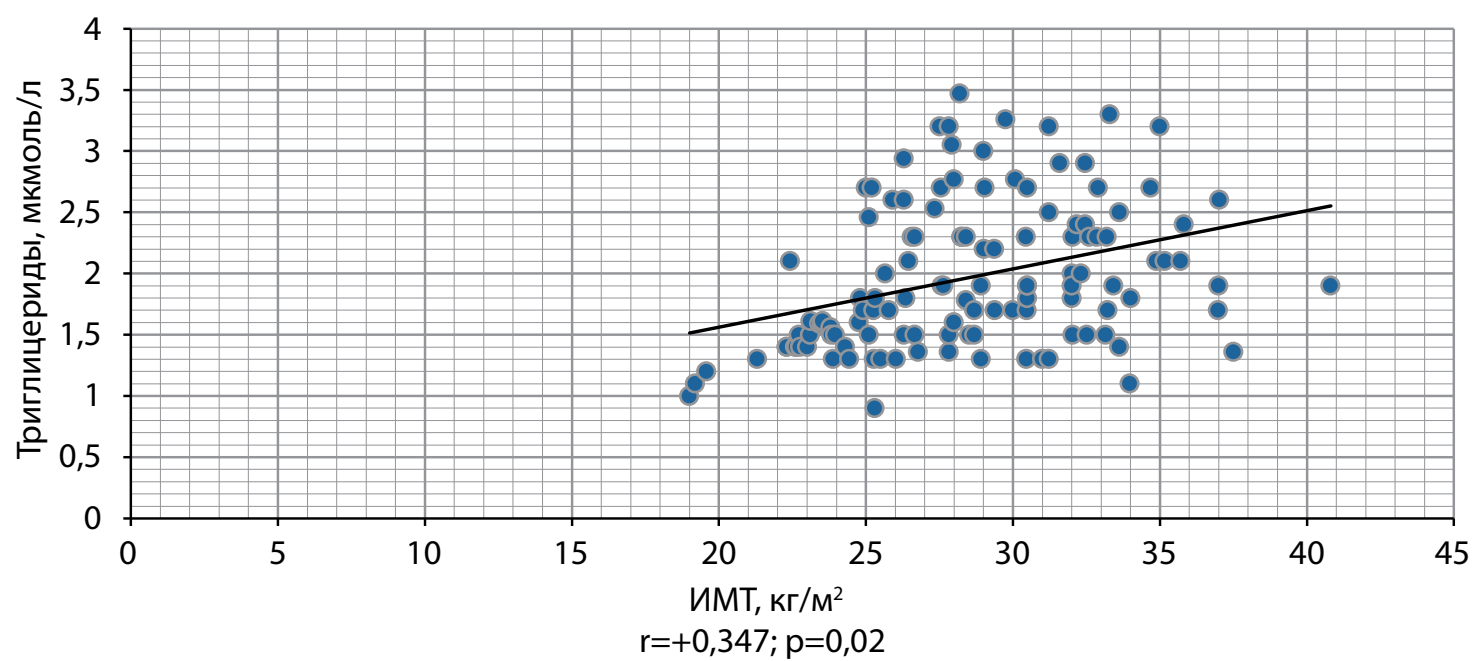

Рисунок 3. График корреляционной зависимости между уровнем триглицеридов сыворотки крови и индексом массы тела. 
Дислипидемия среди пациенток с ожирением и СПКЯ (2-я группа) встречалась чаще по сравнению с пациентками с ожирением $\left(46,6 \%\right.$ vs $22,2 \% ; X^{2}=6,364$ соответственно); также получены различия между 1-й и контрольной группами (22\% vs 9,3\%; $X^{2}=7,514$ соответственно) (см. рис. 1). Средний уровень ТГ, ХС ЛПНП у пациенток с СПКя (2-я группа) оказался выше значений в 1-й группе (женщин с ожирением без СПКЯ), но статистически значимых различий не выявлено $(p=0,06)$; получены значимые различия между 2-й и контрольной группами $(p=0,03)$. Средние концентрации лПВП в сыворотке крови у пациенток с ожирением без СПКЯ были сопоставимы со средними уровнями ЛПВП контрольной группы $(p=0,06) ;$ получены значимые различия между 1-й и 2-й группой $(p=0,04)$ и 2-й группой и группой контроля $(p=0,003)$. Таким образом, наиболее выраженные нарушения липидного спектра крови, проявляющиеся высоким уровнем атерогенных фракций ХС и низким содержанием антиатерогенных ЛПВП, выявлены у пациенток с СПКЯ и ожирением, что закономерно подтверждается более высокими значениями КА, который оказался выше, чем в контрольной группе $(2,01 \pm 0,16$ vs $4,06 \pm 0,183 ; p=0,03)$.

Пациенты с ожирением и СПКЯ имели повышенный уровень СРБ по сравнению с пациентками контрольной группы $(4,10 \pm 0,45$ мг/л vs 0,94 $\pm 0,33$ мг/л соответственно). Уровни СРБ положительно коррелировали с ИМТ $(r=0,404 ; p=0,003)$, соотношением ОТ/ОБ $(r=0,406$; $p=0,003)$ лПНП $(r=0,406 ; p=0,003)$, ТГ $(r=0,306 ; p=0,002)$, фибриногеном $(r=0,706 ; p=0,001)$ и имели отрицательную корреляцию с ЛПВП ( $r=-0,405 ; p=0,0002)$.

Наиболее высокие средние уровни мочевой кислоты выявлялись в группе пациенток с ожирением и СПКЯ $(p=0,001)$. Здесь же значимо чаще выявлялась гиперурикемия (26,6\% vs $10,1 \% ; X^{2}=11,31$ соответственно) (см. рис. 1). Уровень мочевой кислоты отрицательно коррелировал с уровнем ФСГ ( $r=-0,309 ; p=0,03)$, положительно связан с уровнем тестостерона $(r=0,315 ; \mathrm{p}=0,03)$ и с ИМТ $(r=0,296 ; p=0,04)$ и ОТ/ОБ $(r=0,319 ; p=0,03)$.

Наиболее высокие средние уровни индекса HOMA-IR и АМГ были выявлены во 2-й группе, что оказалось значимо выше, чем в остальных $(p=0,002)$.

\section{Дополнительные результаты исследования}

Дефицит витамина D обнаружен у 13,2\% (18 пациенток), недостаточность - у 22,7\% (31 пациентка). Выявлены корреляции между уровнем 25(OH)D и показателями ИМТ, ФСГ, ЛГ ( $r=0,234 ; p=0,030 ; r=0,218 ; p=0,04$; $\mathrm{r}=-0,231 ; \mathrm{p}=0,03$ соответственно). Наиболее высокие средние уровни печеночных трансаминаз выявлены в группе с СПКЯ и ожирением, однако статистически значимые различия с 1 группой получены только для АЛТ $(p=0,045)$. Наиболее высокие уровни тестостерона, ГСПГ и ИСА оказались во 2-й группе по сравнению с контрольной ( $p=0,001 ; p=0,047 ; p=0,002$ соответственно). АМГ положительно коррелировал с уровнями ЛГ $(\mathrm{r}=0,232$; $p=0,03)$, тестостерона $(r=0,212 ; p=0,03)$, с величиной отношения ОТ/ОБ ( $r=0,261 ; p=0,01)$, инсулинорезистентностью $(r=0,273 ; p=0,02)$, отрицательно - с ФСГ $(r=-0,312$; $p=0,002)$, уровнем глюкозы $(r=-0,302 ; p=0,03)$ и инсулина натощак $(r=-0,297 ; p=0,03)$.
По данным УЗИ органов малого таза, у пациенток с ожирением и СПКЯ объем каждого яичника составил в среднем $12,66 \pm 1,78$ см³ $^{3}$ по всей толще обоих яичников обнаруживались множественные фолликулы (более 12 на одном срезе, диаметром до 3-10 мм). У женщин с ожирением и в контрольной группе средний объем

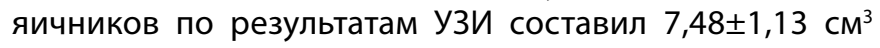
и 7,39 $\pm 1,45$ см $^{3}$ соответственно, признаков поликистоза не выявлено.

\section{Нежелательные явления}

В ходе проведенного исследования нежелательные явления в виде любых медицинских событий (болезней, травм, незапланированных оперативных вмешательств и др.) отсутствовали.

\section{ОБСУЖДЕНИЕ}

Резюме основного результата исследования

Таким образом, в нашем исследовании у женщин с ожирением в сочетании с СПКЯ выявлена высокая частота метаболических нарушений, сопряженных с нарушением фертильности, повышением риска развития атеросклероза и СС3. Обнаружено, что у пациенток с ожирением в сочетании с СПКЯ по сравнению с пациентками без СПКЯ статистически значимо чаще встречались повышение средних уровней СРБ, частоты гиперхолестеринемии, дислипидемии, гиперурикемии, инсулинорезистентности, артериальной гипертензии (АГ), висцерального ожирения.

\section{Обсуждение основного результата исследования}

Индекс атерогенности может повышаться под влиянием яичниковых андрогенов, о чем свидетельствуют полученные нами корреляции между уровнем тестостерона и ОХС. Статистически значимо более высокие уровни индекса НОМА, а также средние уровни инсулина подтверждают роль инсулинорезистентности в патогенезе СПКЯ. Современные авторы подчеркивают наличие нарушений липидного обмена пациенток с СПКЯ в связи с наличием у них гиперандрогении, на фоне которой развивается висцеральное ожирение [10]. По мнению исследователей, при СПкЯ гиперандрогения в 50-60\% случаев сочетается с ожирением и в 70-75\% случаев с гиперинсулинемией $[9,10]$. Висцеральное ожирение вызывает гиперинсулинизм, ИР, нарушение толерантности к глюкозе, что способствует развитию нарушений метаболизма липопротеидов (ЛП) $[1,2,11]$. Так, на фоне развившейся ИР инсулин действует как атерогенный гормон, способствующий увеличению синтеза ТГ и ЛПОНП печенью, ингибированию элиминации этих ЛП из кровеносного русла, что сопровождается увеличением уровня ТГ и ускорением превращения в кровотоке лПОНП в наиболее атерогенные частицы - ЛПнП, а также замедлением образования ЛПВП $[1,2,12]$.

Выявленная в нашей работе статистически значимо более высокая частота гиперурикемии в группе с СПКя объяснима в первую очередь характерной для данной группы пациенток гиперандрогенией. Установлено, что свободный тестостерон положительно связан с концентрацией мочевой кислоты в здоровой 
женской популяции. Более высокие концентрации мочевой кислоты у мужчин по сравнению с женщинами подтверждают возможную корреляцию между андрогеном и мочевой кислотой [13]. Наши результаты также подтвердили, что тестостерон положительно коррелировал с уровнем мочевой кислоты. В некоторых исследованиях выявлено снижение уровня мочевой кислоты после лечения антиандрогенными препаратами у женщин с СПКЯ [14]. В экспериментах с участием лабораторных животных андрогены повышали уровень мочевой кислоты в сыворотке крови, индуцируя печеночный метаболизм пуриновых нуклеотидов и усиливая метаболизм пурина в почках [15]. Результаты этих исследований показали, что метаболизм пурина может быть целью гормонального воздействия; вместе с тем, для подтверждения этой гипотезы необходимы дополнительные исследования.

В нашем исследовании у женщин с СПКЯ отмечались статистически значимо более высокие уровни СРБ и фибриногена по сравнению с группой без СПКЯ. Согласно современным данным, это может указывать не только на высокую вероятность возникновения атеросклероза, но и на повышенный риск тромботических осложнений [16]. По мнению современных авторов, при СПКЯ имеется триада: гиперинсулинемия, гиперандрогения и низкодифференцированное воспаление, действующие вместе в порочном круге патофизиологии СПКЯ $[1,15,16]$.

Полученная нами статистически значимо более высокая частота АГ в группе пациенток с СПКЯ и ожирением находит подтверждение в работах зарубежных исследователей $[1,16]$. Повышенный риск развития АГ при СПКЯ может быть связан с инсулинорезистентностью, гиперинсулинемией и гиперандрогенемией, которые нарушают эндотелий-зависимые механизмы вазодилатации, вызывая гипертрофию сосудистой стенки [17]. Кроме того, у женщин с СПКЯ имеет место повышение маркеров сердечно-сосудистого риска, таких как гиперхолестеринемия, дислипидемия, повышенный уровень СРБ, что также согласуется с полученными нами данными $[4,6,14]$. В этой связи стоит отметить крупное когортное исследование с участием 2301 пациентки, в котором оценивалась частота СС3 у женщин с СПКЯ в течение 20 лет [18]. Согласно полученным данным, более чем у четверти женщин с СПКЯ к возрасту 65 лет развились ИМ или стенокардия [19-21]. Аналогичные данные были получены по результатам недавно проведенного 13-летнего исследования, где выявлено, что женщины с СПКЯ в возрасте $\leq 40$ лет имели скорректированный более высокий риск развития АГ (ОР 2,08; 95\% доверительный интервал (ДИ) 1,0-3,9) и метаболического синдрома (OP 1,81; 95\% ДИ 1,1-2,9). Частота висцерального ожирения была также выше у женщин с СПКЯ в возрасте $\leq 40$ лет по сравнению со здоровыми женщинами группы контроля [19]. Развитие метаболических и сердечно-сосудистых факторов риска у женщин молодого возраста с СПКЯ подчеркивается в исследовании А.Ю. Бегловой и соавт. [20]. В работах П.В. Поповой и соавт. частота СД 2 типа и АГ оказалась достоверно выше в группе больных СПКЯ, однако по частоте встречаемости ИБС различий не оказалось [21].
Обсуждение дополнительных результатов исследования

Уровень АМГ у пациенток с ожирением и СПкЯ оказался статистически значимо выше, чем в группе с ожирением без СПКЯ. Это обусловлено тем, что повышение продукции АМГ яичниками при СПкЯ вызвано не только увеличением числа фолликулов, его вырабатывающих, но и увеличением выработки АМГ гранулезой этих фолликулов $[11,12]$. При висцеральном ожирении гиперинсулинемия стимулирует биосинтез андрогенов в текастромальных клетках яичника, нарушая нормальный рост и созревание фолликулов, снижается уровень АМГ. Поэтому в нашем исследовании уровень АМГ в группе с ожирением без СПКЯ оказался статистически значимо ниже, чем в группе с СПКЯ и контрольной группе $[1,10,11]$. Согласно современным данным, избыточный вес ассоциирован со снижением количества овуляторных циклов, а также со снижением эффективности протоколов индукции овуляции [1, 10-12].

Полученные в нашем исследовании повышенные уровни печеночных трансаминаз у женщин с ожирением в сочетании с СПКЯ согласуются с данными современной литературы [1, 11, 22]. Так, проведенный недавно метаанализ выявил более высокую распространенность неалкогольной жировой болезни печени (НАЖБП) среди женщин с СПКЯ. Наличие НАЖБП ассоциировано с высоким уровнем андрогенов в сыворотке крови в дополнение к ожирению и инсулинорезистентности [22]. Ряд перекрестных исследований демонстрирует значительное увеличение распространенности НАЖБП у молодых женщин с СПКЯ независимо от избыточного веса/ожирения и других особенностей метаболического синдрома, эти женщины с большей вероятностью имеют более тяжелые формы НАЖБП (неалкогольный стеатогепатит, фиброз и цирроз печени) [23]. Накопленные данные свидетельствуют о том, что стеатогепатоз может усугублять печеночную и системную инсулинорезистентность, способствуя повышению высвобождения провоспалительных, прокоагулянтных и профиброгенных медиаторов, играющих важную роль в патофизиологии СПКЯ [10, 22, 23].

Статистически значимых различий в средних уровнях витамина D между исследуемыми группами в нашей работе не выявлено, хотя в ряде исследований предполагалось существование возможной связи между низким уровнем витамина D и CПКЯ $[23,24]$. Вместе с тем нами выявлена статистически значимая отрицательная корреляция между уровнем витамина D и величиной ИМТ, а также высокая частота дефицита и недостаточности витамина D. Существует гипотеза, согласно которой дефицит витамина D, приводя к дефициту половых гормонов, способствует нарушению соотношения жирозапасающих и жиросжигающих гормонов. Имеющееся и прогрессирующее ожирение уменьшает уровень циркулирующего в крови витамина D за счет повышенного захвата жировой тканью и снижения скорости гидроксилирования в печени за счет стеатогепатоза [25]. Это формирует своеобразный «порочный круг патогенеза», характерный для инсулинорезистентности и метаболического синдрома $[24,25]$. Сегодня существуют доказательства, что низкий уровень витамина D может рассматриваться как независимый предиктор формирования 
и прогрессирования ожирения, ведущего к инсулинорезистентности и СПКЯ [26]. Доказано, что витамин D может влиять на чувствительность тканей к инсулину с помощью двух механизмов: либо непосредственно стимулируя экспрессию рецепторов инсулина в клетках, либо косвенно, за счет увеличения внеклеточного кальция, необходимого для инсулиноопосредованных внутриклеточных процессов в инсулинзависимых тканях $[24,26]$. С другой стороны, витамин D находится в тесной взаимосвязи с половыми стероидами, которые также влияют на экспрессию генов инсулинового рецептора и его чувствительность [24-26]. Некоторыми авторами высказывалось предположение, что прием витамина D может способствовать стабилизации менструального цикла и увеличивать частоту наступления беременности у женщин с СПКЯ [26]. Таким образом, дефицит витамина D может быть фактором, дополнительно способствующим развитию различных аспектов метаболического синдрома, которые обычно наблюдаются у женщин с СПКЯ.

Ограничения исследования

Ограничением нашего исследования является небольшой объем выборки.

\section{ЗАКЛЮЧЕНИЕ}

В нашем исследовании у пациенток с ожирением в сочетании с СПКЯ статистически значимо чаще, чем у женщин с ожирением, выявлялись АГ и метаболические нарушения, такие как дислипидемия, висцеральное ожирение, инсулинорезистентность, гиперинсу- линемия, гиперурикемия, что суммарно увеличивает кардиоваскулярный риск. Учитывая, что модифицируемые факторы риска СС3 потенциально обратимы, необходимо применять существующие знания о кардиоваскулярном риске в клинической практике ведения женщин с СПКЯ в сочетании с ожирением. На первый план в данном случае выходят немедикаментозные способы лечения, такие как полноценная физическая активность и правильное питание с целью снижения массы тела. Кроме того, важен скрининг традиционных факторов риска СС3, что рекомендовано многими международными организациями.

\section{ДОПОЛНИТЕЛЬНАЯ ИНФОРМАЦИЯ}

Источники финансирования. Работа выполнена по инициативе авторов на базе Клиники ФГБОУ ВО КубГМУ Минздрава РФ.

Конфликт интересов. Авторы декларируют отсутствие явных и потенциальных конфликтов интересов, связанных с содержанием настоящей статьи.

Участие авторов. Болотова Е.В. - разработка протокола исследования, интерпретация результатов, внесение правок в рукопись; Дудникова А.В. - анализ и интерпретация полученных результатов, подготовка рукописи, внесение правок в рукопись; Крутова В.А. - разработка протокола исследования, контроль и координация проведения исследования; Просолупова Н.С.- сбор материала, анализ результатов, подготовка рукописи, внесение правок в рукопись. Все авторы одобрили финальную версию статьи перед публикацией, выразили согласие нести ответственность за все аспекты работы, подразумевающую надлежащее изучение и решение вопросов, связанных с точностью или добросовестностью любой части работы.

\section{СПИСОК ЛИТЕРАТУРЫ | REFERENCES}

1. $\mathrm{WHO}$ «Obesity and overweight». Fact sheet 2018; 311 . Доступно по http://www.who.int/mediacentre/factsheets/fs311/en/. Ссылка действительна на 21.03.20. [WHO «Obesity and overweight». Fact sheet 2018; 311 [cited 21.03.20]. Available from: http://www.who.int/mediacentre/factsheets/fs311/en/]

2. Gambineri A, Laudisio D, Marocco C, et al. Obesity Programs of nutrition, Education, Research and Assessment (OPERA) group. Female infertility: which role for obesity? Int J Obes Suppl. 2019;9(1):65-72. doi: https://doi.org/10.1038/s41367-019-0009-1

3. Болотова Е.В., Самородская И.В., Комиссарова И.М. Взаимосвязь индекса массы тела и абдоминального ожирения на примере сельской популяции Краснодарского края // Ожирение и метаболизм. - 2016. - Т. 13. - №1. C. 25-29. [Bolotova EV, Samorodskaya IV, Komissarova IM. The relationship between body mass index and abdominal obesity on the example of a rural population of the Krasnodar territory. Obesity and metabolism. 2016;13(1):25-29. (In Russ.)]. doi: https://doi.org/10.14341/omet2016125-29

4. Дедов И.И., Мельниченко Г.А., Шестакова М.В., и др. Лечение морбидного ожирения у взрослых // Ожирение и метаболизм. - 2018. - T. 15. - №. 1 - C. 53-70. [Dedov II, Melnichenko GA, Shestakova MV, et al. Morbid obesity treatment in adults. Obesity and metabolism. 2018;15(1):53-70. (In Russ.)]. doi: https://doi.org/10.14341/OMET2018153-70

5. Синдром поликистозных яичников в репродуктивном возрасте (современные подходы кдиагностике и лечению). Клинические рекомендации (протокол лечения). Министерство здравоохранения РФ. Письмо от 10 июня 2015 г. N 15-4/10/2-2814. [Polycystic ovarian syndrome in reproductive age (contemporary approaches to diagnosis and treatment). Clinical recommendations (treatment Protocol). Ministry of health of the Russian Federation. Letter dated June 10, 2015 N 15-4/10/2-2814. (In Russ.)].
6. De Leo V, Musacchio MC, Cappelli V, et al. Genetic, hormonal and metabolic aspects of PCOS: an update. Reprod Biol Endocrinol. 2016;14(1):38. doi: https://doi.org 10.1186/s12958-016-0173-х.

7. Пигарова Е.А., Рожинская Л.Я., Белая Ж.Е., и др. Клинические рекомендации Российской ассоциации эндокринологов по диагностике, лечению и профилактике дефицита витамина D у взрослых // Проблемы Эндокринологии. 2016. - T. 62. - №4. - C. 60-84. [Pigarova EA, Rozhinskaya LYa, Belaya ZhE, et al. Russian Association of Endocrinologists recommendations for diagnosis, treatment and prevention of vitamin D deficiency in adults. Problems of endocrinology. 2016;62(4):60-84. (In Russ.)]. doi: https://doi.org/10.14341/probl201662460-84

8. Ожирение. Клинические рекомендации 2020. Утверждены Минздравом РФ 17.02.2021. [Ozhirenie. Klinicheskie rekomendacii 2020. Utverzhdeny Minzdravom RF 17.02.2021 (In Russ.)].

9. Артериальная гипертензия у взрослых. Клинические рекомендачии. 2020. [Arterial'naya gipertenziya u vzroslyh. Klinicheskie rekomendacii. 2020. (In Russ.)].

10. Jeanes YM, Reeves S. Metabolic consequences of obesity and insulin resistance in polycystic ovary syndrome: diagnostic and methodological challenges. Nutr Res Rev. 2017;30(1):97-105. doi: https://doi.org 10.1017/S0954422416000287

11. Goodman NF, Cobin RH, Futterweit W, et al. American Association of Clinical Endocrinologists (AACE); American College of Endocrinology (ACE); Androge Excess and PCOS Society. American association of clinical endocrinologists, american college of endocrinology, and androgen excess and pcos society disease state clinical review: guide to the best practices in the evaluation and treatment of polycystic ovary syndrome - part 2. Endocr Pract. 2015;21(12):1415-26. doi: https://doi.org 10.4158/EP15748.DSCPT2 
12. Дурманова А.К., Отарбаев Н.К. Антимюллеров гормон как показатель репродуктивного здоровья женщин с ожирением и сопутствующим синдромом поликистозных яичников // Терапевтический архив. - 2016. - Т. 88. — №12. - C. 41-44. [Durmanova AK, Otarbaev NK. Anti-Muller hormone as an indicator of reproductive health of women with obesity and concomitant polycystic ovary syndrome. Therapeutic archive. 2016;88(12):41-44. (In Russ.)]. doi: https://doi.org10.17116/terarkh2016881241-44

13. Sova H, Unkila-Kallio L, Tiitinen A, et al. Hormone profiling, including anti-Müllerian hormone (AMH), for the diagnosis of polycystic ovary syndrome (PCOS) and characterization of PCOS phenotypes. Gynecol Endocrinol. 2019;35(7):595-600 doi: https://doi.org 10.1080/09513590.2018.1559807

14. Mu L, Pan J, Yang L, et al. Association between the prevalence of hyperuricemia and reproductive hormones in polycystic ovary syndrome. Reprod Biol Endocrinol. 2018;16(1):104. doi: https://doi.org/10.1186/s12958-018-0419-x

15. Arslan E, Gorkem U, Togrul C. Is There a Relationship Between Vitamin D Deficiency Status and PCOS in Infertile Women? Geburtshilfe Frauenheilkd. 2019;79(7):723-730. doi: https://doi.org 10.1055/a-0871-6831

16. Macut D, Mladenović V, Bjekić-Macut J, et al. Hypertension in Polycystic Ovary Syndrome: Novel Insights. Curr Hypertens Rev. 2020;16(1):55-60. doi: https://doi.org/10.2174/1573402115666190531071422

17. Крутова В.А., Дудникова А.В. Взаимосвязь нарушений репродуктивной функции и высокого кардиоваскулярного риска // Акушерство и гинекология. - 2019. №5. - C. 19-24. [Krutova VA, Dudnikova AV. Relationship between reproductive dysfunction and high cardiovascular risk. Obstetrics and Gynecology. 2019;5:19-24. (In Russ.)] doi: https://doi.org/10.18565/aig.2019.5.19-24

18. Mani H, Levy MJ, Davies MJ, et al. Diabetes and cardiovascular events in women with polycystic ovary syndrome. A 20-year retrospective cohort study. Clin Endocrinol. 2013;78(6):926-934. doi: https://doi.org/10.1111/cen.12068
19. Behboudi-Gandevani S, Ramezani Tehrani F, et al. Cardiometabolic risks in polycystic ovary syndrome: long-term populationbased follow-up study. Fertil Steril. 2018;110(7):1377-1386. doi: https://doi.org/10.1016/j.fertnstert.2018.08.046

20. Беглова А.Ю, Елгина С.И. Метаболические и сердечно-сосудистые риски у женщин репродуктивного возраста с синдромом поликистозных яичников // Мать и дитя в Кузбассе. 2018. - №4. - C. 44-49. [Beglova AYu, Elgina SI. Metabolicheskie i serdechno-sosudistye riski u zhenshchin reproduktivnogo vozrasta s sindromom polikistoznykh yaichnikov. Mat' i ditya v Kuzbasse. 2018;4:44-49. (In Russ.)].

21. Попова П.В., Горелова И.В., Гринева Е.В. Синдром поликистозных яичников и кардиоваскулярный риск // Артериальная гипертензия. - 2018. - Т. 24. - №6. - C. 654-665. [Popova PV, Gorelova IV, Grineva EN. Polycystic ovary syndrome and cardiovascular risk. Arterial Hypertens. 2019;24(6):654-665. (In Russ.)]. doi: https://doi.org/10.18705/1607-419X-2018-24-6-654-665

22. Rocha ALL, Faria LC, Guimarães TCM, et al. Non-alcoholic fatty liver disease in women with polycystic ovary syndrome: systematic review and meta-analysis. J Endocrinol Invest. 2017; 40(12):1279-1288. doi: https://doi.org 10.1007/s40618-017-0708-9

23. Petta S, Ciresi A, Bianco J, et al. Insulin resistance and hyperandrogenism drive steatosis and fibrosis risk in young females with PCOS. PLOS One. 2017;12(11):e0186136. doi: https://doi.org10.1371/journal.pone.0186136

24. Krul-Poel YHM, Koenders PP, Steegers-Theunissen RP, et al. Vitamin $D$ and metabolic disturbances in polycystic ovary syndrome (PCOS): A cross-sectional study. PLoS One. 2018; 4;13(12):e0204748. doi: https://doi.org 10.1371/journal.pone.0204748

25. Shi XY, Huang AP, Xie DW, Yu XL. Association of vitamin D receptor gene variants with polycystic ovary syndrome: a meta-analysis. BMC Med Genet. 2019;20(1):32. doi: https://doi.org 10.1186/s12881-019-0763-5.

26. Voulgaris N, Papanastasiou L, Piaditis G, et al. Vitamin D and aspects of female fertility. Hormones (Athens). 2017;16(1):5-21. doi: https://doi.org 10.14310/horm.2002.1715

\section{ИНФОРМАЦИЯ ОБ АВТОРАХ [AUTHORS INFO]:}

* Дудникова Анна Валерьевна, к.м.н. [Anna V. Dudnikova, MD, PhD]; адрес: 350010, Россия, г. Краснодар, ул. Зиповская, д. 4/3 [address: 4/1 Zipovskaya str., Krasnodar, 350010, Russia]; ORCID: https://orcid.org/0000-0003-2601-7831; eLibrary SPIN: 7480-1992; e-mail: avdudnikova@yandex.ru

Болотова Елена Валентиновна, д.м.Н., профессор [Elena V. Bolotova, MD, PhD, professor]; ORCID: https://orcid.org/0000-0001-6257-354X; eLibrary SPIN: 4322-9985; e-mail: bolotowa_e@mail.ru Крутова Виктория Александровна, д.м.н., профессор [Victoria A. Krutova, MD, PhD, professor]; ORCID: https://orcid.org/0000-0002-9907-7491; eLibrary SPIN: 1673-1155; e-mail: klinika@bagk-med.ru Просолупова Наталия Сергеевна [Natalia S. Prosolupova, MD]; ORCID: https://orcid.org/0000-0003-1888-3991; eLibrary SPIN: 1192-0437; e-mail: bill.berries@mail.ru

*Автор, ответственный за переписку / Corresponding author.

\section{ЦИТИРОВАТЬ:}

Болотова Е.В., Дудникова А.В., Крутова В.А., Просолупова Н.С. Частота и структура метаболических нарушений у женщин с ожирением в сочетании с синдромом поликистозных яичников // Ожирение и метаболизм. - 2021. T. 18. — №3. — C. 254-262. doi: https://doi.org/10.14341/omet12374

\section{TO CITE THIS ARTICLE:}

Bolotova EV, Dudnikova AV, Krutova VA, Prosolupova NS. The frequency and structure of metabolic disorders in obese women in combination with polycystic ovary syndrome. Obesity and metabolism. 2021;18(3):254-262. doi: https://doi.org/10.14341/omet12374 